\title{
COVID-19 targets the right lung
}

\author{
Jingwen $\mathrm{Li}^{1+}$, Xiaxia $\mathrm{Yu}^{2+}$, Shaoping $\mathrm{Hu}^{3}$, Zhicheng $\mathrm{Lin}^{4}$, Nian Xiong ${ }^{1,5^{*}}$ and $\mathrm{Yi} \mathrm{GaO}^{2^{*}}$ (D)
}

Previous imaging studies of COVID-19 suggested that bilateral lungs be affected [1]. In this study, we noticed a side-preference of lung lesions in COVID-19. The lesions on the right lung were significantly larger and developed faster than those on the left. Moreover, the level of right-over-left preference of lung injury was significantly correlated with the potential need for intensive care and inpatient mortality.

Pulmonary lesions were imaged by a total of 253 high resolution computed tomographic (CT) chest scans of 103 COVID-19 patients at Wuhan Red Cross Hospital. Of the 103 patients with a median (interquartile range (IQR)) age of $63(47-70)$ years, 57 were males, 41 were in the intensive care unit (ICU), and 64 were deceased during hospitalization. At the time of the study, only 1 patient was still in the ICU. The median (IQR) length of known hospital stay was 16 (10-24) days.

Two independent assessments of the images were conducted. First, one physician visually evaluated the level of injury on either side of the lung and provided percentage-based semiquantitative scores on the injury of the lung for either side (0: no, $1:<5 \%, 2: 6-25 \%$, 3: $26-50 \%, 4: 51-75 \%, 5:>75 \%$ injury). Second, an automatic deep learning-based algorithm [2] extracted the three-dimensional (3D) features of all lesions that were validated by physicians. Then, quantitative volumetric analysis and comparisons were carried out for rightversus left-side lesions' volumes and their growth speed.

As a result, 70\% (31/103) CT scans showed that the lesion volume of the right lung was larger than that of the left lung. Figure 1 shows a typical case in panels a and b. In addition to the first-time $\mathrm{CT}$ of each patient, we studied 253 longitudinal CT images of 103 patients (2-3 time points/patient).

In order to facilitate the discussion below, here we denoted Delta $(\Delta)$ as the difference of the volume of the lesion on the right lung and that of the lesion on the left lung. A potential association between the age and $\Delta$ was also analyzed. The results showed that the older the patient, the larger the $\Delta\left(\chi^{2}\right.$ test for linear terms, $\left.F=11.9466, P<0.0001\right)$ (Fig. 2a). Moreover, the non-survivors had bigger $\Delta$ than the survivors (one-sided $t$ test, $P<0.0001$ ) (as shown in Fig. 1c). Smooth curves were fitted for $\Delta$ and a log risk ratio was used for death. These results indicated the lowest risk ratio when the $\Delta$ was around 0 , and the differences between the left and right lungs were minimal. The risk for death increased with larger $\Delta(P=0.013$, Fig. $2 b)$. In order to understand which side was correlated with a severe form of the disease, Fisher's extract test was used to compare the fatality risk of patients with smaller lesions on the left side to those with larger lesions on the left. The results suggested that patients with large lesions on the right lung be at a high mortality risk during hospitalization $(\mathrm{OR}=2.662$, $P$ value $=0.0252$ (Fig. 2c, d).

\footnotetext{
*Correspondence: nianxiong@hust.edu.cn; gaoyi@szu.edu.cn

${ }^{+}$Jingwen Li and Xiaxia Yu contributed equally to this work.

'Department of Neurology, Union Hospital, Tongji Medical College,

Huazhong University of Science and Technology, Wuhan, Hubei, China

${ }^{2}$ School of Biomedical Engineering, Health Science Center, Shenzhen

University, Shenzhen, Guangdong, China

Full list of author information is available at the end of the article
} 

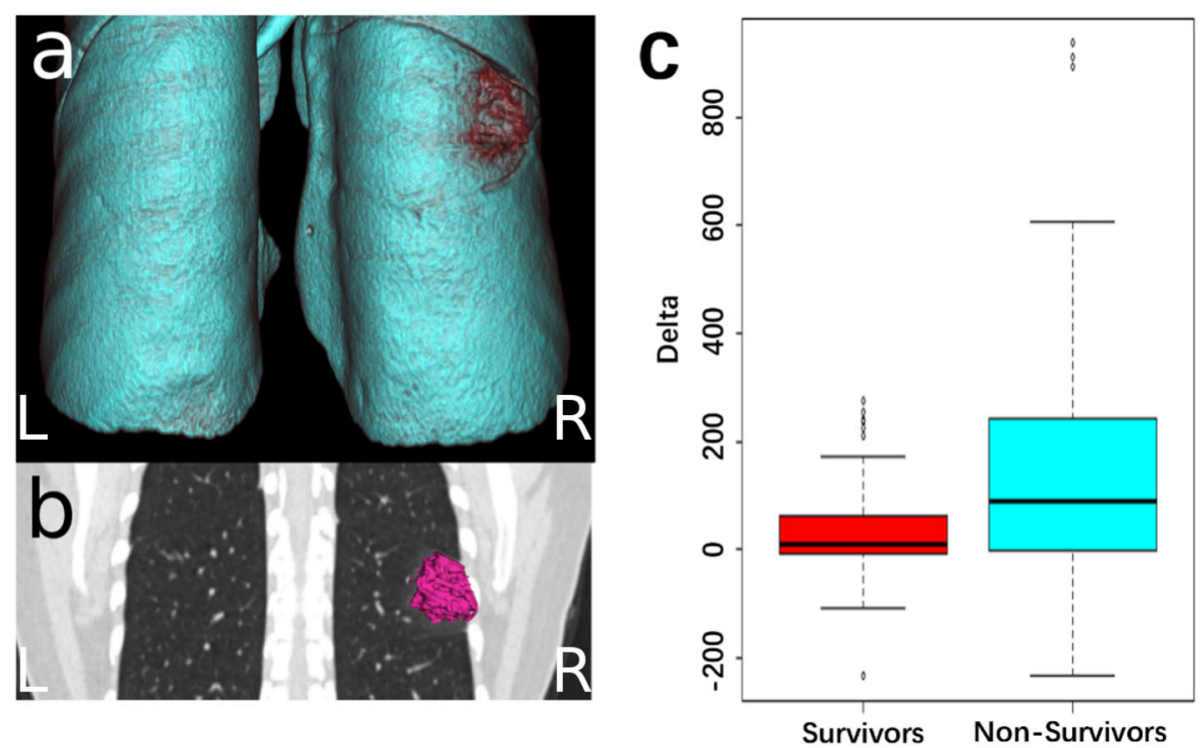

Fig. 1 a $3 D$ renders the highlights of a lesion on the right lung (view from posterior). b 3D extraction of the lesions. c Comparison of $\Delta$ in nonsurviviors and survivors (one-sided $t$ test, $P<0.0001$ )

Different diseases have different origins of lesions. For instance, foreign bodies are likely to be aspirated in the right bronchus because of the short, wide, and straight path [3]. Tuberculosis prefers the right upper lobes [4], which might be attributed to the oxygen distribution ratio. In COVID-19, the right sidepreference was consistent with a reported autopsy result that the right lung was subject to hemorrhage [5]. These results draw care attention to the right lung in this novel pneumonia.

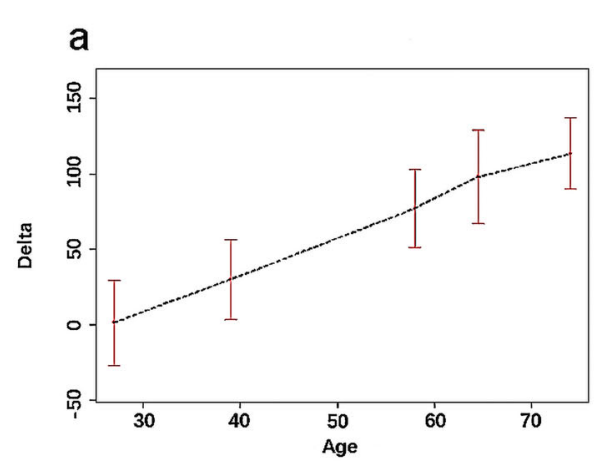

C

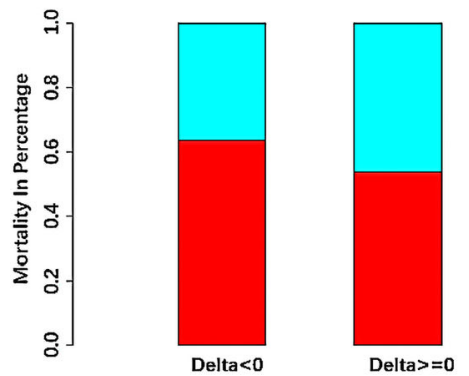

b

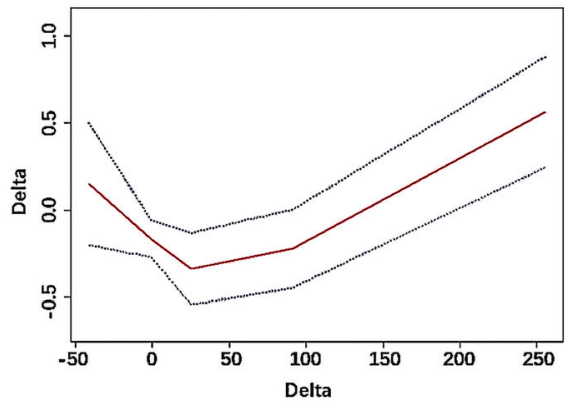

d

\begin{tabular}{|l|l|l|l|}
\hline RLS-LLS & Death & Alive & Total \\
\hline \multirow{2}{*}{0} & $\begin{array}{l}51 \\
(63.75 \%)\end{array}$ & $\begin{array}{l}29 \\
(36.25 \%)\end{array}$ & $\begin{array}{l}(100 \%) \\
\end{array}$ \\
\hline$\geqslant 0$ & $\begin{array}{l}93 \\
(53.76 \%)\end{array}$ & $\begin{array}{l}80 \\
(46.24 \%)\end{array}$ & $\begin{array}{l}121 \\
(100 \%)\end{array}$ \\
\hline
\end{tabular}

Fig. 2 Statistical analysis. a Correlation between age and delta. Age was categorized into five groups. b Association between $\Delta$ and relative death ratio. c Comparison of mortality in patients with $\Delta<0$ and $\Delta \geq 0$. Mortality rate of non-survivors was shown in cyan; mortality rate of survivors was shown in red. $\mathbf{d}$ Contingency table for $\Delta$ and mortality 


\section{Acknowledgments}

Not applicable.

\section{Authors' contributions}

$Z L, N X$, and $Y G$ helped in the conception and design of the study. $J \mathrm{~L}$ and $\mathrm{SH}$ contributed to data collection. $X Y$ and $Y G$ contributed to data analysis and draft writing. YG and NX contributed to the final version of the manuscript before submission. All authors commented on previous versions of the manuscript and read and approved the final manuscript.

\section{Funding}

This study was supported by grants 2016YFC1306600 (to NX) and 2018 YFC1314700 (to NX) from the National Key R\&D Program of China, grants 81873782 (to NX) from the National Natural Science Foundation of China, grant 2016CFB624 from Natural Science Foundation of Hubei Province (to NX), grant 2017050304010278 from The Youth Science and Technology Morning Light Program of Wuhan City (to NX), 2018 Hubei Medical Research Project WJ2019F030 (to NX), 2018 Wuhan Medical Research Project S201802140011 (to NX), 2018 Wuhan Young and Middle-aged Medical Talent Program (to NX), 2017 Hubei provincial Party Committee Organization Department of Second Batch of Hubei Youth Elite Development Plan (to NX), National Natural Science Foundation of China No. 61601302 (to YG), Department of Education of Guangdong Province No. 2017KZDXM072 (to YG), Shenzhen Peacock Plan No. KQTD2016053112051497 (to YG), and Startup funding for Youth Faculty by Shenzhen University grant 2018009 (to XY).

\section{Availability of data and materials}

The datasets used and/or analyzed during the current study are available from the corresponding author on reasonable request.

\section{Ethics approval and consent to participate}

Wuhan Red Cross Hospital provided the waiver of consent before participation in the study.

\section{Consent for publication}

Not applicable.

\section{Competing interests}

The authors declare that they have no competing interests.

\section{Author details}

'Department of Neurology, Union Hospital, Tongji Medical College, Huazhong University of Science and Technology, Wuhan, Hubei, China. ${ }^{2}$ School of Biomedical Engineering, Health Science Center, Shenzhen University, Shenzhen, Guangdong, China. ${ }^{3}$ Department of Radiology, Wuhan Red Cross Hospital, Wuhan, Hubei, China. ${ }^{4}$ Harvard Medical School, Belmont, MA 02478, USA. ${ }^{5}$ Medical Treatment Expert Group for Covid-19, Wuhan Red Cross Hospital, Wuhan, Hubei, China.

Received: 30 April 2020 Accepted: 27 May 2020

Published online: 15 June 2020

\section{References}

1. Shi H, Han X, Jiang N, et al. Radiological findings from 81 patients with COVID-19 pneumonia in Wuhan, China: a descriptive study. Lancet Infect Dis. 2020;20(4):425-34. https://doi.org/10.1016/S1473-3099(20)30086-4.

2. He, J., Yu, X., Cai, C. \& Gao, Y. MCMC Guided CNN Training and Segmentation for Pancreas Extraction. arXiv:2003.03938 (2020).

3. Chen $\mathrm{CH}$, et al. Foreign body aspiration into the lower airway in Chinese adults. Chest. 1997;112(1):129-33.

4. Zhou L, Han L, Xie LZ. Characterizations in digital chest X-ray of 459 pulmonary tuberculosis. In Modern Med. 2019;35(22):3507-9 (in Chinese).

5. Luo, W.; Yu, H.; Gou, J.; Li, X.; Sun, Y.; Li, J.; Liu, L. Clinical Pathology of Critical Patient with Novel Coronavirus Pneumonia (COVID-19). Preprints 2020020407 (2020).

\section{Publisher's Note}

Springer Nature remains neutral with regard to jurisdictional claims in published maps and institutional affiliations. 